\title{
Electrically Conductive High-Performance Thermoplastic Filaments for Fused Filament Fabrication
}

\author{
Ozge Kaynan ${ }^{\mathrm{a}, \mathrm{e}, * *}$, Alptekin Yildız ${ }^{\mathrm{b}, \mathrm{e}, * *}$, Yunus Emre Bozkurt ${ }^{\mathrm{c}, \mathrm{e}}$, Elif Ozden Yenigun ${ }^{\mathrm{d}, \mathrm{e}, *}$, Hulya Cebeci ${ }^{\mathrm{b}, \mathrm{e}, *}$ \\ ${ }^{a}$ Department of Textile Engineering, Istanbul Technical University, Istanbul, 34469, Turkey \\ ${ }^{b}$ Department of Aeronautical Engineering, Istanbul Technical University, Istanbul, 34469, Turkey \\ ${ }^{c}$ Department of Defence Technologies, Istanbul Technical University, Istanbul, 34469, Turkey \\ ${ }^{d}$ School of Design, Textiles, Royal College of Art, London, SW7 2EU, United Kingdom \\ ${ }^{e}$ ITU Aerospace Research Center, Istanbul Technical University, Istanbul, 34469, Turkey
}

\begin{abstract}
Conductive polyetherimide (PEI)-based filaments can fill the gap between the design and manufacturing of functional and structural components through additive manufacturing. This study systematically describes the fabrication of carbon nanotube (CNTs)-reinforced PEI filaments, complemented by a custom-built extrusion process facilitating low weight fraction of nanomaterials. Neat PEI and CNTs/PEI filaments at different CNTs fractions ranging from 0.1 to 7 wt. \% were fabricated. Supported by morphology analysis, the rheological percolation was found to be higher $(0.25$ wt. \% CNTs/PEI) than electrical percolation $(0.1 \mathrm{wt}$. \% CNTs/PEI) since the system reached an electrical percolation within the formation of a continuous conductive path at lower CNTs loadings. With the $7 \mathrm{wt}$ \% CNTs loading, the highest electrical conductivity of CNTs/PEI filaments was reported as $2.57 \times 10^{-1} \mathrm{~S} / \mathrm{cm}$. A $55 \%$ enhancement in tensile modulus was achieved when 5 wt. \% CNTs were introduced, but in a trade-off in elongation at break ca. $65 \%$.
\end{abstract}

Keywords: Polymer Nanocomposite, Polyetherimide, Carbon Nanotubes, Fused Filament Fabrication

\section{Introduction}

Additive manufacturing (AM) offers the implementation of a multi-material approach to achieve functionality for integrated structures with design freedom and complex structural manufacturing capability. These material systems can offer significant advantages in highly demanding fields such as aerospace, electronics and biomedical science. Creating functional parts and pieces by using polymer feedstocks in AM has limitations; however, the advantages outweigh these challenges. One of the main challenges of using polymers in AM is the limited off-the-shelf feedstocks. Therefore, multi-functional and material solutions can be presented either by using functionally graded materials [14], feedstocks of low melting and engineering polymers [5-7] or by tuning the properties by adding nanomaterials such as carbon nanotubes (CNTs) and graphene [8, 9] in an integrated structure.

From prototyping to final assembly, Fused Filament Fabrication (FFF) has gained momentum through several researchers efforts to revolutionize these technologies in the context of both the machine and the material realms. FFF is a layer-by-layer deposition method of building objects in any geometry with guided nozzles that melt and extrude filaments. The feedstock materials for extrusion range from low-performance polymers such as acrylonitrile butadiene styrene (ABS), polycarbonates (PC), polyamide 6 (PA 6), polylactic acid (PLA) and polyphenylene sulfide (PPS) to high-performance polymers such as polyether ether ketone (PEEK), polyaryletherketone (PAEK) and polyetherimide (PEI). In order to explore a range of new possibilities, such as 3D printed circuitry, touch sensors, electromagnetic interference (EMI) and radiofrequency (RF) shielding, as well as thermal protection systems (TPS) in composite applications, the question of whether nanomaterials can tailor conventional or high-performance polymers used in 3D

\footnotetext{
${ }^{*}$ Corresponding authors

** These authors contributed equally to this work

Email addresses: elif .ozden-yenigun@rca.ac.uk (Elif Ozden Yenigun), hulya.cebeci@itu.edu.tr (Hulya Cebeci)
} 
printing filaments was investigated. However, only a small number of polymers, such as PEEK, PEI and PAEK and their derivatives, meet the requirements for solvent and flame resistance and the necessary overall strength in such demanding fields to bring functionality to the overall structure with reinforcing strategies.

Therefore, it is feasible to select and process a polymer that has the required certifications and reinforce it with several nanofillers from carbon-based fillers such as CNTs and graphene, or with very aggressive fillers (short carbon fiber and glass fiber). CNTs are found to be useful in reinforcing composite structures by adding functionality. To date, researchers have been seeking new polymeric solutions that range from rigid plastics to rubber-like materials, whether these are insulating or conductive, to be processed with off-the-shelf 3D printers.

To assess conductivity contribution of CNTs to thermoplastics, Dul et al. [10] focused on the mixing of CNTs up to $8 \mathrm{wt}$. $\%$ in ABS matrix and then fabricated filaments with a diameter of $1.7 \mathrm{~mm}$. The resistivity of these highly loaded 8 wt. \% CNTs reinforced with ABS filaments measured as low as $0.55 \Omega . \mathrm{cm}$. Moreover, it was also reported that $6 \mathrm{wt}$. \% CNTs/ABS filaments exhibited an approximately 12\% increase in tensile strength compared to the standard ABS filaments. It is also important to note that even though stiff CNTs have made a substantial contribution in terms of optimizing the performance of moderate polymers such as ABS, this still cannot satisfy the necessary requirements for filaments to be used in advanced components. Therefore, when strength and chemical and thermal durability are design concerns, the odds are in favour of high-performance polymers.

Among the high-performance polymers, PEI, also known by its commercial name, ULTEM, brings an exciting combination of properties, such as high glass transition temperature $\left(217^{\circ} \mathrm{C}\right)$, and excellent mechanical properties (tensile strength of $c a .90 \mathrm{MPa}$ ), and has aerospace certifications which relate to flammability, smoke, and toxicity. However, a minimal number of studies have been conducted that focus on melt processing and/or the FFF applications of functional PEI filaments where process challenges arise from its high viscosity and elevated extrusion temperatures. Chuang et al. [11] reported a study assessing ULTEM 9085, ULTEM 1000 and 10 wt. \% chopped carbon fiberreinforced ULTEM 1000 filaments. Through the extrusion process, a $6 \mathrm{~mm}$ length of carbon fibers was reduced to 2-3 mm, resulting in a significant decrease in aspect ratio.

Recently, Berretta et al. [12] have produced CNTs/PEEK FFF filaments at 1 and $5 \mathrm{wt}$. \% of CNTs concentrations. The results showed that $5 \mathrm{wt}$. \% CNTs/PEEK filaments reached the ultimate tensile strength of $105 \mathrm{MPa}$, while standard PEEK filaments reached $100 \mathrm{MPa}$. Even though CNTs were relatively well distributed in the PEEK matrix, their effect in toughening was limited. All these efforts still cannot articulate material processing challenges in highperformance polymers associated with nanomaterial inclusion, or the way in which the CNT network formation and enhancement capabilities within mechanical properties and electrical conductivity differ, by reporting discussions of PEIs' rheological behavior. Polyakov et al. [13] investigated the effect of various loadings of carbon nanofibers (vapor-grown carbon nanofiber with an external diameter of 100nm and a length ranging between 20-200 $\mu \mathrm{m}$ ) in ULTEM 1000, specifically to identify the mechanical properties of FFF printed parts. Incorporating 1 wt. \% of carbon nanofiber improved the tensile strength of neat ULTEM 1000 by $8 \%$, as well as modulus by $11 \%$, while keeping the elongation at break at the same level, around 9\%. Ghose et al. [14] studied the addition of different nanofillers containing CNTs, carbon fibers and expanded graphite, with the content ranging from 5 to 40 wt \% into ULTEM 1000 , and observed that all these nanofibers aligned through extrusion direction and assisted thermal conduction. In a recent study, Wu et al. [15] investigated the thermal and flammability characteristics of multi-functional PEI-based composite filaments made of hollow glass microspheres, nanoclay and non-halogenated flame-retardant additive combinations. They reported that the formulation, consisting of $10 \mathrm{wt}$. \% glass bubbles, $5 \mathrm{wt}$. \% nanoclay and 10 wt. \% flame-retardant additives, exhibited a $10.7 \%$ improvement in char yield and a $52 \%$ reduction in heat release capacity compared to neat PEI.

Our study attempts to engineer melt-processing of PEI for CNTs/PEI filament fabrication by revealing the effect of the inclusion of CNTs in both the extrusion process and the final characteristics of conductive CNTs/PEI filaments. It began by designing the extrusion equipment itself and then optimizing the process parameters to achieve effective dispersion and distribution of CNTs in PEI to avoid any additives and compounding agents. Functionalization of CNTs was also out of the question, due to the additional cost and/or process, and as a result PEI-based conductive 3D printer filament was developed by using industrial-grade low-cost CNTs for advanced applications. To the best of our knowledge, this is the first work that articulates the fabrication and characterization of CNT-reinforced PEI filaments for 3D printing. Neat PEI and CNTs/PEI filaments with various concentrations from 0.1 to $7 \mathrm{wt}$. \% CNTs were produced by consecutive twin- and single-screw extrusion processes, where CNTs were fed through a side feeder during twin-screw extrusion. We highlighted the critical role of the dispersion state of CNTs and their relative concentration, 
along with the challenges in the melt processing of PEI. Thermal, morphological, electrical, rheological, and mechanical testing were carried out to reveal the shear-driven dispersion and reinforcing mechanism of CNTs in depth. The results revealed that the incorporation of $5 \mathrm{wt}$. \% CNTs improved the electrical conductivity of PEI filaments by 14 times and increased the tensile modulus by $55 \%$ compared to neat PEI matrix, with a trade-off in elongation at break $\%$.

\section{Materials and Methods}

\subsection{Materials}

Polyetherimide (Poly (bisphenol A anhydride-co-1,3-phenylenediamine, PEI) granules (Tecotek®EI20 NL) were purchased from Eurotech (ULTEM 1000, $\mathrm{C}_{37} \mathrm{H}_{24} \mathrm{O}_{6} \mathrm{~N}_{2}$, Density: $1.27 \mathrm{~g} / \mathrm{cm}^{3}$, MW: $592 \mathrm{~g} / \mathrm{mol}$, glass transition temperature $\left.(T g): 217^{\circ} \mathrm{C}\right)$. CNTs were purchased from Nanokomp (industrial-scale multi-walled CNTs with carbon purity: min. $90 \%$, diameter: $5-15 \mathrm{~nm}$, length: $1.5-2 \mu \mathrm{m})$. All chemicals were used without further purification.

\subsection{Melt processing of PEI and CNTs/PEI Nanocomposite Filaments}

Before the melt processing, PEI granules were first conditioned in a vacuum oven at $150^{\circ} \mathrm{C}$ for eight hours to stabilize weight loss associated with abundant water molecules. A single-screw extruder that has a screw diameter of $12 \mathrm{~mm}$ with length $(\mathrm{L})$ to diameter $(\mathrm{D})$ ratio $\mathrm{L} / \mathrm{D}=22$, designed and manufactured by Kökbir Import\&Export, was used to fabricate neat PEI filaments with a $60 \mathrm{rpm}$ screw speed at a drawing ratio of 20. Single-screw extruder, as illustrated in Fig. 1 consists of six different barrels, which can be controlled in each zone temperature. A typical single-screw design employs feed, transition, and metering stages. The screw pitch of the feed stage is 12/12 (pitch $[\mathrm{mm}] /$ diameter of the screw $[\mathrm{mm}]$ ), and the pitch gradually decreases in transition until the metering stage, in which the screw pitch narrows down to 7/12. By decreasing the screw pitch, enough pressure and drag forces were created for the melt flow through the die, and the channel depth was kept the same throughout the screw.

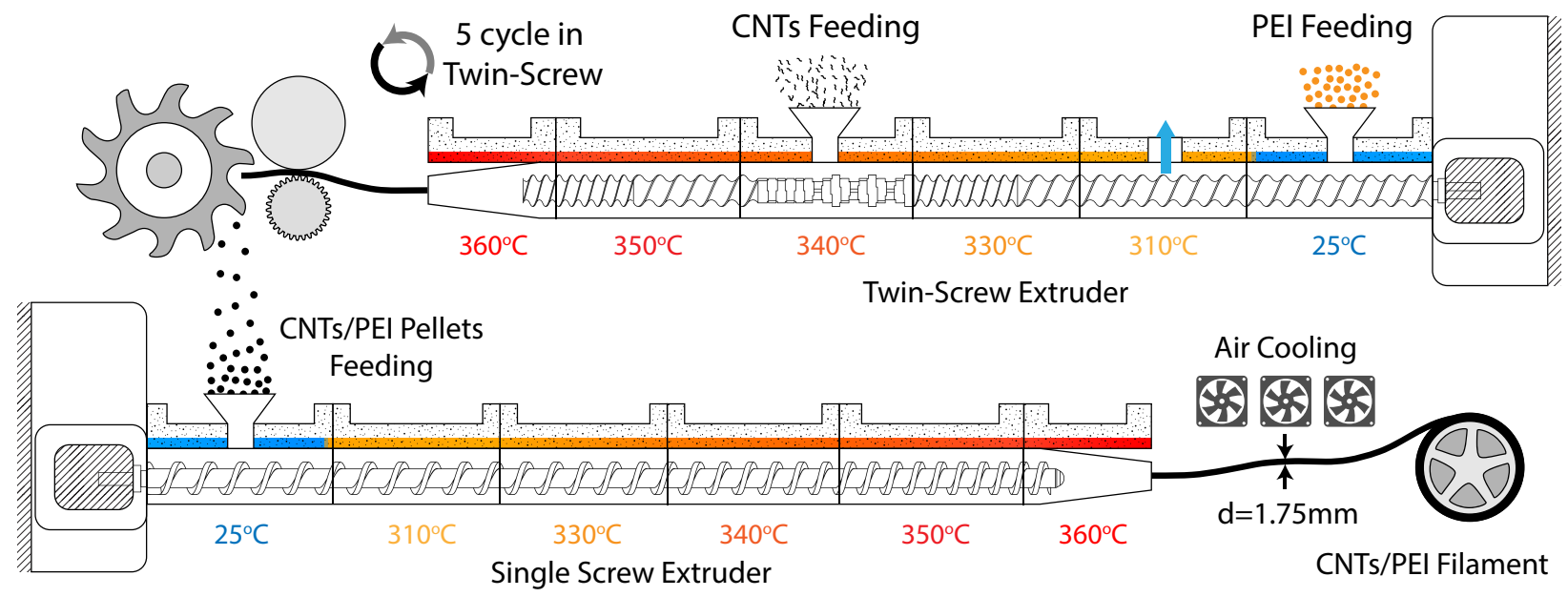

Figure 1: Illustration depicting the melt processing of neat PEI and CNTs/PEI filaments by twin-screw extrusion accompanied by single-screw extrusion until filament winding.

The CNTs/PEI granules were fabricated by using a co-rotating twin-screw with a screw diameter of $12 \mathrm{~mm} L$ to $D$ ratio, $L / D=22$, where CNTs were fed through a side feeder at the targeted CNTs concentration. Then the CNTs/PEI granules were fed into a single-screw extruder to achieve CNTs/PEI filaments. The screw configuration of these custom-built extruders, as illustrated in Fig. 1 was mainly designed to process highly viscous materials such as PEI and PEEK at a barrel temperature of $310-360^{\circ} \mathrm{C}$ from hopper to die, accompanied by downstream accessories, including a counter-current air dryer, rotating knife and drawing unit. The extruders, having six different mixing zones, create a wide range of thermomechanical stresses [16], all of which had to be precisely monitored. In the twinscrew extruder, to transport unmelted and/or partially melted polymer granules, 16/12 conveying elements were placed 
in the first two barrels of the twin-screw. Subsequent 16/12 and 10/12 conveying elements enabled the complete melting of the polymer, where heat-activated mobile polymer chains become disentangled. Sequential kneading blocks with $45 / 5 / 18$ and 90/5/18 (staggering angle $\left[{ }^{\circ}\right] /$ number of tooth/length [mm]) were used in the next barrel, to promote the homogeneous dispersion and distribution of nanofillers in the molten polymer matrix. 16/12 and 10/12 conveying elements are employed in the fifth and sixth barrels respectively to elevate pressure through the die.

Twin-screw extrusion was employed for five cycles to avoid the agglomerations of CNTs; subsequently, these CNTs/PEI nanocomposites were cut by a rotating knife and then granulated. The number of cycles for CNTs/PEI was determined with a parametric study of the characterization of the material property/number of cycles based on electrical conductivity measurements. To produce 3D printer filaments with a nominal diameter of $1.75 \mathrm{~mm}$, a singlescrew extruder, as depicted in Fig. 1] was used for both neat PEI and CNTs/PEI filaments. CNTs were fed through a side feeder to achieve 2, 5, and 7 wt. \% CNTs/PEI granules. To reveal the percolation threshold of CNTs/PEI filaments, 0.1, 0.25, and 0.5 wt. \% CNTs/PEI filaments were fabricated by diluting 2 wt. \% CNTs/PEI granules, as also seen in [17]. In both neat PEI and CNTs/PEI nanocomposite filaments, a series of barrel temperatures were used and set to $310,330,340,350$, and $360^{\circ} \mathrm{C}$, respectively. The neat PEI and CNTs/PEI filaments were wound and quenched under air. Melt processing details can be found in the supporting video. In addition, 3D printing video can be found in supporting files.

\subsection{Characterization Techniques}

Differential scanning calorimetry (DSC) measurements were performed on a 4000 Perkin Elmer DSC instrument (Waltham, MA, USA). Approximately $9 \mathrm{mg}$ samples were weighed and sealed in an aluminum crucible. Each sample was heated from $50^{\circ} \mathrm{C}$ to $350^{\circ} \mathrm{C}$ under an inert $\mathrm{N}_{2}$ atmosphere (flow of $20 \mathrm{~mL} / \mathrm{min}$ ) at a heating rate of $10^{\circ} \mathrm{C} / \mathrm{min}$ to clear the thermal history of the samples. Then, they were cooled down from $350^{\circ} \mathrm{C}$ to $50^{\circ} \mathrm{C}$ under fast cooling and followed by a reheating step up to $350^{\circ} \mathrm{C}$ at a heating rate of $10^{\circ} \mathrm{C} / \mathrm{min}$. The second heating scan was used for further analysis. The $T g$ was determined by measuring mid-points of the peaks.

The thermal stability of neat PEI and CNTs/PEI filaments was determined using an Exstar TG/DTA 7200Thermogravimetric Analysis (TGA) (RT Instruments, Woodland, CA, USA). All samples were heated from $30^{\circ} \mathrm{C}$ to $800^{\circ} \mathrm{C}$ at a rate of $10^{\circ} \mathrm{C} / \mathrm{min}$ in an inert $\mathrm{N}_{2}$ flow of $2 \mathrm{~mL} / \mathrm{min}$. Difference Thermogravimetry (DTG) thermographs were drawn from the first derivative of TGA weight loss raw data with respect to temperature. The thermal decomposition temperatures $(T d)$ of each sample were evaluated by the peak points of the DTG thermographs.

The cryo-fractured cross-sections of neat PEI and CNTs/PEI filaments were investigated by Field Emission Scanning Electron Microscope (FE-SEM), (SEM, LEO 1530VP), operating at $15 \mathrm{keV}$. A thin layer of the gold-palladium alloy was sputtered using a Quorum evaporator with a covering time of 45 seconds for neat PEI and CNT/PEI samples to avoid charging during electron irradiation. The samples were then placed into a vacuum chamber, and the electron beam was focused on the cross-section of the fractured edge.

Transmission electron microscopy (TEM) analysis was carried out to reveal the microscopic features and dispersion state regarding the CNTs wt. \% and the changes in the aspect ratio of the CNTs. A Multi-beam focused ion beam, (JEOL JIB-4601 MultiBeam FIB-SEM system, equipped with EDS and RAITH Elphy Quantum FIB/SEM Nanolithography Systems), was used to prepare thin slides of nanocomposites with a thickness of 100nm. Then JEOL JEM-ARM200CFEG UHR-TEM (equipped with STEM, Cs-corrected STEM, EDS, Gatan Quantum GIF, and Digital CCD Camera) device operated at $200 \mathrm{kV}$ was used for TEM analysis.

Dynamic rheological measurements of neat PEI and CNTs/PEI filaments were conducted using an oscillatory rheometer (Anton Paar, Modular Compact Rheometer MCR 102) equipped with plate-plate geometry with a diameter of $25 \mathrm{~mm}$. Before the experiment, all the samples, which were in the form of granules, were conditioned in a vacuum oven at $150^{\circ} \mathrm{C}$ for eight hours. The samples, with $25 \mathrm{~mm}$ diameter and $2 \mathrm{~mm}$ gap thickness, were placed between the preheated plates at $360^{\circ} \mathrm{C}$ and kept for five minutes to transform the solid granules into the melt phase before each frequency sweep test was carried out under $\mathrm{N}_{2}$ atmosphere. The complex shear modulus (storage modulus and loss modulus) was recorded as a function of angular velocity in a range between 0.1 and $500 \mathrm{rad} / \mathrm{s}$, to evaluate a linear viscoelastic domain.

The electrical conductivities of neat PEI and CNTs/PEI filaments were measured by a Four-Point Probe measuring system FPP 470 (Entek Electronics, Turkey) at room temperature, as shown in Fig. S1. Each specimen was $2.5 \mathrm{~cm}$ long and $0.85 \mathrm{~mm}$ thick, and before measurement the specimen surfaces were polished using sandpapers with 400 , 800,1200 , and 2500 grits in a sequence to reduce roughness. 
Tensile testing was performed at room temperature by using a Zwick Roell Z100 testing machine with a load capacity of $100 \mathrm{kN}$, using single-bollard, self-tightening type grids and a manual extensometer. The tensile properties of both neat PEI and 5 wt. \% CNTs/PEI filaments, such as their ultimate strength, fracture strength and tensile modulus, were determined at a crosshead speed of $5 \mathrm{~mm} / \mathrm{min}$, and each test was repeated at least five times. In accordance with ASTM 3822D, $60 \mathrm{~cm}$-long specimens with $110 \mathrm{~mm}$ gauge length were used.

\section{Results and Discussion}

The complexity in the melt processing of PEI might be eased or disrupted by the addition of CNTs, associated with heat-driven local ordering of PEI and CNTs. Thus, thermal analysis traces the effect of CNTs on the melt state and provides insight into kinetic and thermodynamic transitions driven by these new domains. These transitions, and how they changed with the incorporation of CNTs, were detected by DSC analysis, as revealed in 2 a. PEI is an amorphous thermoplastic polymer. Therefore we focused only on $\mathrm{Tg} \mathrm{Tg}$ and searched for the effect of CNTs on this temperature range. Fig. 2 demonstrated that the existence of CNTs in the PEI matrix did not lead to a notable change in $T g$. Even with the addition of up to $7 \mathrm{wt}$. \% CNTs, the $T g$ of CNTs/PEI was found to be similar to the $T g$ of neat PEI, where $T g$ of neat PEI, 5 wt. \% and 7 wt. \% CNTs/PEI filaments were reported as 216,217 , and $214{ }^{\circ} \mathrm{C}$, respectively.
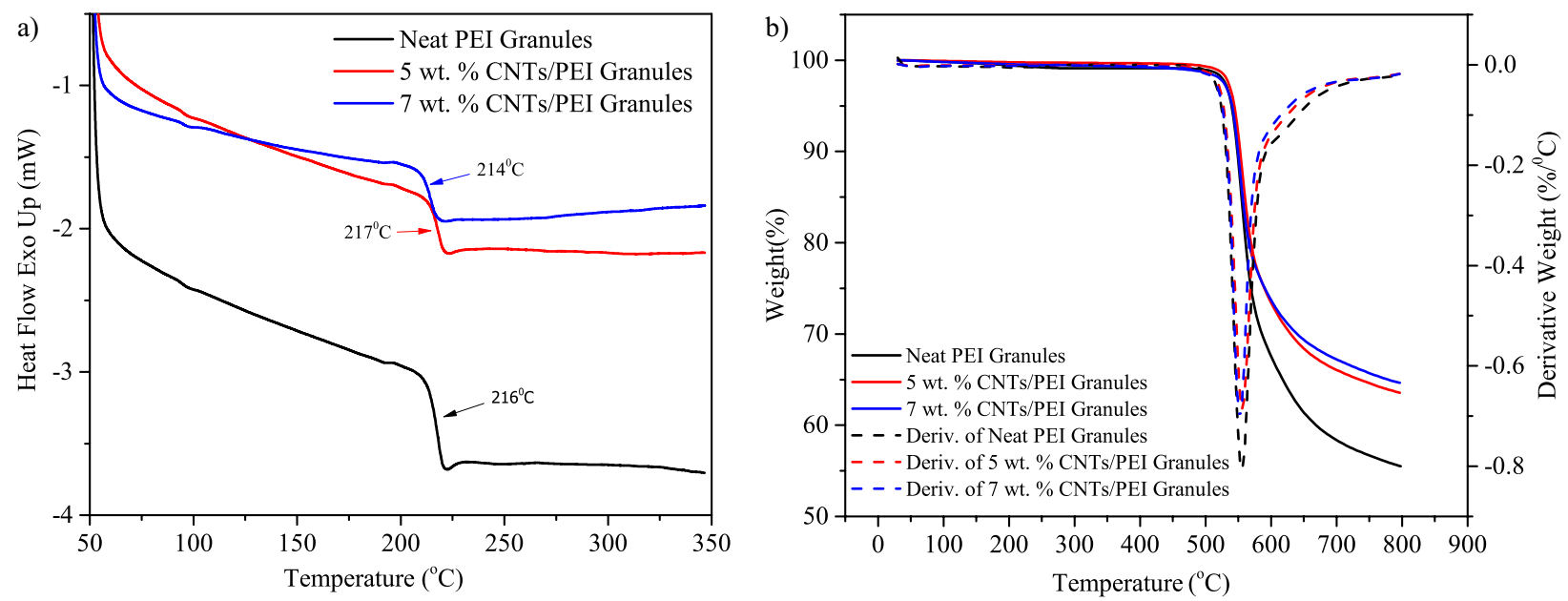

Figure 2: a) DSC analysis of neat PEI and 5 and 7 wt. \% of CNTs/PEI nanocomposite granules. Arrows pointed out $T g$ of each granules and b) TGA analysis of neat PEI and 5 and 7 wt. \% of CNTs/PEI nanocomposite granules, along with the first derivative of mass change (DTG) in the TGA graph.

On the other hand, Liu et al. [18] noted an increased $\mathrm{Tg}$ in CNT-incorporated PEI fabricated by solution mixing techniques, and that $1 \mathrm{wt}$. \% CNTs addition to PEI restricted the polymer chain mobility through enhanced interfacial adhesion, eventually shifting the $T g$. CNTs might increase or depress the $T g$, depending on their attractive/repulsive interaction between surrounding polymers and interfacial adhesion, as well as their relative concentration [19, 20]. In most cases, since $T g$ is a kinetic phenomenon, the presence of CNTs in the polymer matrix does not lead to a significant $T g$ difference in polymer matrix nanocomposites [12, 21], which can be attributed either to weak polymer/filler interaction [22] or to a reduced CNTs aspect ratio suffering from twin-screw extrusion cycles [23]. In fact, a consistent $T g$ range in nanocomposites has practical advantages in $3 \mathrm{D}$ printing applications. It therefore keeps the balance between multi-functionality and printing system stability without bringing additional processing challenges, which is critical for printing nanocomposite structures similar to original polymers [24]. A study of Pitchan et al. [25] reported slightly enhanced $T g$ of PEI with the incorporation of plasma and chemical-functionalized CNTs by solvent precipitation and dry mixing, which was attributed to the improved wettability and adhesion of polymer-CNTs which had been restricting the polymer chain mobility. Nevertheless, as in this study, once pristine CNTs were used as reinforcements, there were no significant $T g$ changes noted [10, 12, 21, 26].

Thermal decomposition temperature $\left(T_{d}\right)$ defines the breaking down of chemical bonds in a material by heating, which provides distinctive information on thermal stability derived from the peak point of DTG. Thermal decomposi- 
tion analysis derived from DTG was conducted on neat PEI; 5 and 7 wt. \% of CNTs/PEI filaments from $30^{\circ} \mathrm{C}$ to $800^{\circ} \mathrm{C}$ under $\mathrm{N}_{2}$ atmosphere and the corresponding curves are displayed in Fig. $2 \mathrm{p}$ and also reported in Table 1 . Thermographs of all the samples demonstrated thermal stability up to $450^{\circ} \mathrm{C}$, indicating that no additives and/or solvents were added during the melt processing [27]. The chemical structure of PEI consists of both aromatic and non-aromatic groups [28], and both TGA and DTG thermograms exhibited a characteristic two-step decomposition for each sample, where the scission of the imide group played an essential role in this pyrolysis process. First, mass change related to the non-aromatic unit decomposition of PEI; second, decay can be associated with the decomposition of more stable aromatic groups [29]. Table 1 suggests that the temperature of neat PEI, 5 wt. \% and 7 wt. \% CNTs/PEI filaments, which 5 wt. $\%$ mass loss occurred, were $538^{\circ} \mathrm{C}, 544^{\circ} \mathrm{C}$, and $540^{\circ} \mathrm{C}$ respectively, corresponding to the decomposition of non-aromatic groups in PEI. Through the incorporation of CNTs, a slight increase in $T_{d}$ was seen, which was interpreted from $T_{\text {max }}$ in the DTG thermograph. In accordance, $T_{d}$ of neat PEI filament shifted from $552^{\circ} \mathrm{C}$ to $558^{\circ} \mathrm{C}$ and $555^{\circ} \mathrm{C}$ for $5 \mathrm{wt} . \%$ and $7 \mathrm{wt} . \% \mathrm{CNTs} / \mathrm{PEI}$ filaments, respectively. This finding suggests that the presence of CNTs contributed a slight enhancement in thermal stability. Furthermore, the increase in $T_{d}$ can be associated with good dispersion of CNTs and effective interaction between CNTs and PEI [27]. Since strong interaction through the $\pi-\pi$ bonds hinders the polymer chain mobility and raises the barrier effect, the high thermal conductivity of the CNT facilitates the dissipation of heat inside the composite, which delays the thermal decomposition [20, 27, 30, 31]. At 30 wt. $\%$ loss of the neat PEI, 5 wt. $\%$ and 7 wt. \% CNTs/PEI filaments were 584, 628, and $640{ }^{\circ} \mathrm{C}$, as seen in Table 1. The resultant residual mass at $800^{\circ} \mathrm{C}$ supported the previous finding, where the residue for neat PEI, $5 \mathrm{wt} . \%$ and $7 \mathrm{wt}$. \% CNTs/PEI filaments were $55 \mathrm{wt}$. \%, $63 \mathrm{wt}$. \% and $65 \mathrm{wt}$. \%, respectively. An increase in mass balance correlatively with the concentration of CNTs is attributed to the introduction of a significant amount of carbon from CNTs. Overall, the thermal stability of PEI was increased with the incorporation of CNTs.

Table 1: TGA and DTG results of neat PEI and 5 and 7 wt. \% CNTs/PEI filaments

\begin{tabular}{|c|c|c|c|}
\hline & Neat PEI filament & 5 wt. $\%$ CNTs/PEI filament & 7 wt. \% CNTs/PEI filament \\
\hline$T_{\max }\left({ }^{\circ} \mathrm{C}\right)$ & 552 & 558 & 555 \\
\hline 5 wt. $\%$ loss $\left({ }^{\circ} \mathrm{C}\right)$ & 538 & 544 & 540 \\
\hline 30 wt. \% loss $\left({ }^{\circ} \mathrm{C}\right)$ & 584 & 628 & 640 \\
\hline Residue at $800^{\circ} \mathrm{C}(\mathrm{wt} . \%)$ & 56 & 63 & 65 \\
\hline
\end{tabular}

Fig. 3 demonstrated three micrographs of cryo-fractured neat PEI and CNT/PEI filaments with 5 and 7 wt. \% CNTs. Fig. 3a shows that along with dispersion quality, the neat PEI formation in which local polymer bridging was observed. At the nanoscale level, without applying any surface treatment and functionalization, CNTs were dispersed and distributed homogeneously, and no significant agglomeration was detected in either composition, as revealed in Figs. 3p and 3k. Furthermore, Figs. $3 \mathrm{p}$ and $3 \mathrm{k}$ also demonstrated that CNTs effectively adhered to the PEI matrix, indicating good wettability between them. The fibrillary formation of CNTs in thermoplastic resins, as in our case of PEI, has also been observed by various researchers [10, 17, 25, 26, 32], where the brighter white lines and dots in Figs. $3 \mathrm{~b}$ and $3 \mathrm{r}$ can be identified as the tail end of CNTs. These images pointed out that CNTs were well covered with PEI, and no exact pull-out of them was observed when they were cryo-fractured [10]. To enhance the dispersibility of CNTs in melt polymer, Isayev et al.[32] integrated an ultrasonic treatment unit into a twin-screw extruder which collaboratively works during melt processing, and they achieved homogeneous dispersion of CNTs into PEI. Herein, our proposed process enables the conditions for the dispersal of CNTs straightforwardly, in a similar way to the reference study [32], without using new processes and/or units. As an alternative approach for achieving good dispersion, Yan et al.[23] also performed various extrusion cycles for their single-walled carbon nanotubes/polyacrylonitrile. They observed that increasing this number of process cycles led to a better dispersion of nanotubes in solution; however, the diameter of the single-walled carbon nanotube bundles decreased after the fourth extrusion cycle. Similarly, through high shear forces during twin-screw extrusion with the five cycles in this study, adequate processing conditions were created to facilitate the homogeneous dispersion and distribution of CNTs.

Fig. 4 points out the corresponding TEM images taken at the lowest CNT loading, $0.1 \mathrm{wt}$. \%, and at the highest, 7 wt. $\%$ in CNTs/PEI filaments through increased magnification. As expected, applying five cycles of twin-screw extrusion lowered the length of CNTs from $1.5-2 \mu \mathrm{m}$ to $305 \pm 20 \mathrm{~nm}$. Therefore, to achieve a percolation threshold for an efficient electron transfer, 7 wt. \% CNTs/PEI filament showed a successful network formation with CNTs in 


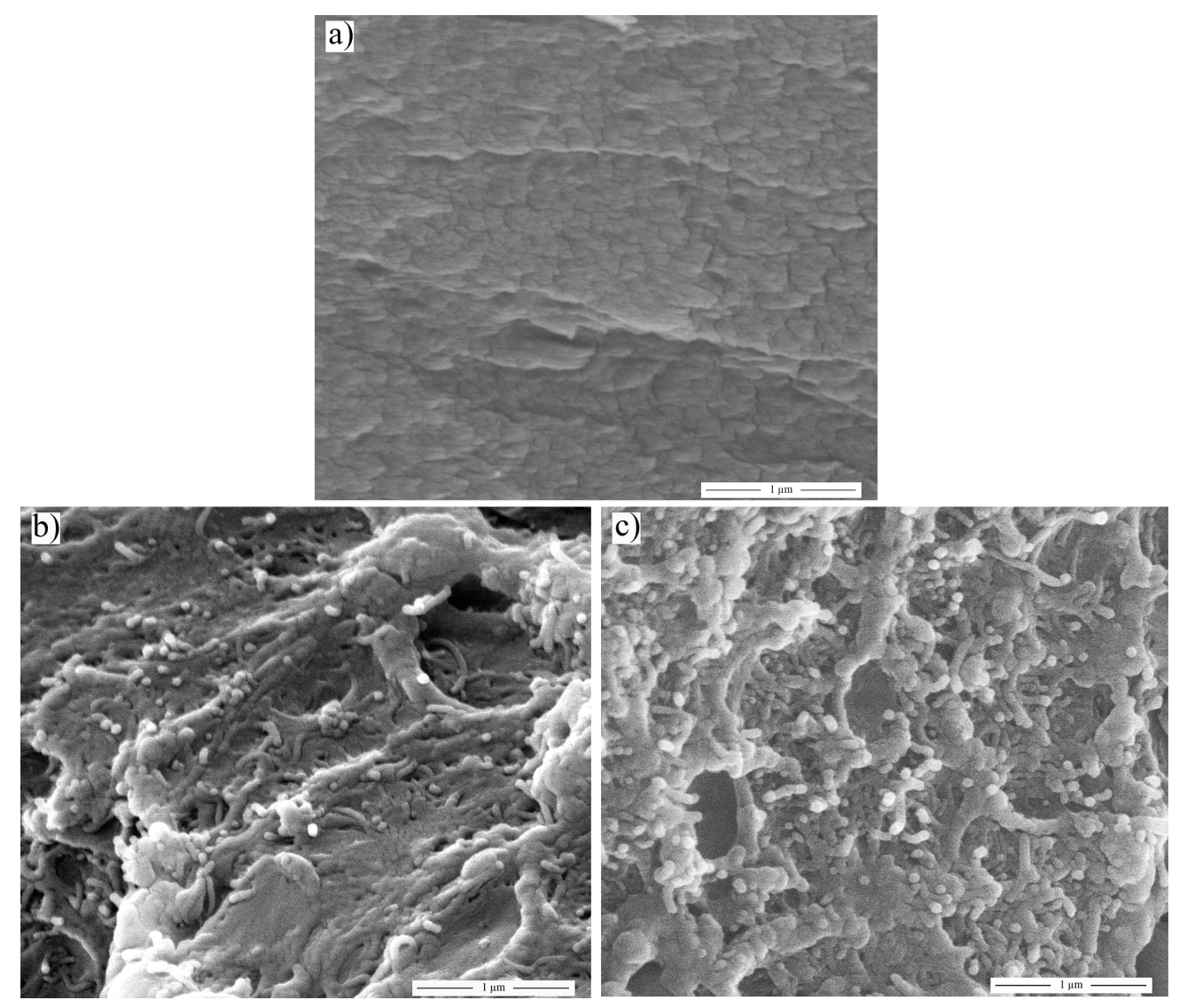

Figure 3: Cross-section SEM images of cryo-fractured a) neat PEI filament, b) 5 wt. \% CNTs/PEI and c) 7 wt. \% CNTs/PEI filament. The white lines were identified as CNTs tails (Fig. $3 \mathrm{~b}$ and c)

contact, while a 0.1 wt. \% concentration was not sufficient (Fig. 4 a and b). Moreover, all the evidence gained from morphological analysis suggested that without CNT aggregates and highly aligned CNTs, effective melt processing was achieved to overcome the critical challenges faced by others in using high-performance viscous polymers [12, 17, 25, 33, 34]. Furthermore, it is also reported that PEI acted as a good host with no additive, solvent or pre-process for CNTs, which can be attributed to the customized design of screw extruders and restricted CNT movement due to its high viscosity [34]. Therefore the successful dispersion and distribution of CNTs in PEI increased the tensile stiffness of the CNTs/PEI filaments and reduced the percolation threshold [17]. Since CNTs were nearby and in contact, the electrical conductivity increased drastically when $7 \mathrm{wt}$ \% CNTs/PEI filament was considered.

All these changes in the aspect ratio of CNTs, when combined with shear stresses enforcing orientation towards extrusion direction, changed the polymer conformations around CNTs and CNT clusters. Therefore, based on experimental observations coupled with theoretical models, to predict the presence of CNTs clustering under extrusion conditions could provide insight into the real state of CNTs in the PEI matrix. J. Li et al. [35] defined a theoretical model based on the average interparticle distance (IPD), which could also apply to CNTs nanocomposites. The model assumed that a nanocomposite is divided into cubic elements with a length of L; each unit has one conductive particle located in the center, and the total number of cubic elements is equal to the total number of particles. The conductive particles could be in the form of either a single CNT with a length of $L L$ and a diameter of $D$ or a CNTs bundle. The real case includes both individual CNTs as well as their agglomerates.

This model suggests two main approaches to estimate the percolation threshold. The first approach describes the state if IPD is less than or equal to $10 \mathrm{~nm}$, which is lower than the length of individual CNTs. The second approach also considers the dispersion state by implementing two dispersion parameters, $\epsilon$, and $\xi$ to reveal how the dispersion state and the aspect ratio of CNTs affects the percolation. The percolation threshold, $P_{c}$, can be defined by the following 

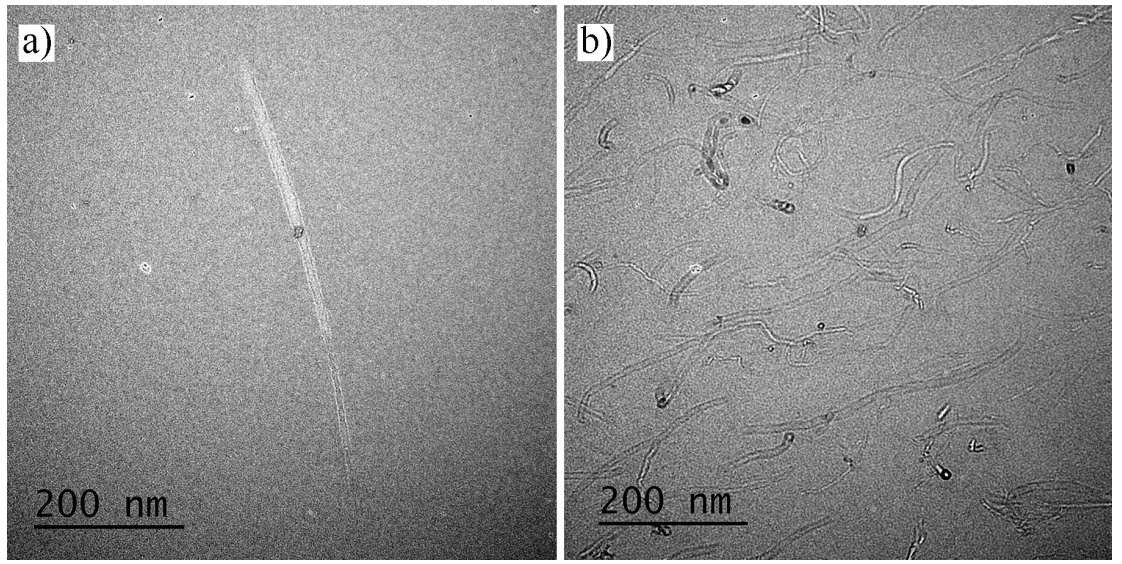

Figure 4: TEM micrographs of a) $0.1 \mathrm{wt}$. \% and b) $7 \mathrm{wt} . \% \mathrm{CNTs} / \mathrm{PEI}$ filaments. The thickness of the samples was $100 \mathrm{~nm}$, prepared by a focused ion beam. The average length of CNTs was $305 \pm 20 \mathrm{~nm}$, measured by Fig $4 \mathrm{~b}$.

equation.

$$
P_{c}=\frac{\xi \epsilon \pi}{6}+\frac{(1-\xi) 27 \pi D^{2}}{4 L^{2}}
$$

where $\epsilon$ is the localized volume content of CNTs in an agglomerate, and $\xi$ is the volume fraction of agglomerated CNTs.

Eq. 11 not only estimates the percolation threshold using experimental values of aspect ratios $(L / D)$ but also provides information about the dispersion state in nanocomposites by giving a range for $\epsilon$ and $\xi$ values, which is later interpreted as CNTs' tendency of clustering.

TEM images in Fig. 4 show that the mean length of CNTs was $c a .305 \pm 20 \mathrm{~nm}$. Therefore, assuming the mean diameter of CNTs was around $10 \mathrm{~nm}$, the aspect ratio of CNTs embedded into nanocomposites can be calculated as 30. Fig. 5 depicts a 3D contour plot derived by Eq. 1 representing the percolation threshold with respect to $\epsilon$ and $\xi$. The percolation threshold range in our study, also referring to electrical percolation in CNTs/PEI filaments, is highlighted as a gray contour in Fig. 5. The weight fractions, wt. \% were converted to volume fractions, vol. \%, using the theoretical density of CNTs, given as $1.8 \mathrm{~g} / \mathrm{cm}^{3}$ [35]. Grey shaded area in Fig. 5 clearly indicates that the ratio of the local volume fraction of CNTs, whether individual or clusters, to the volume fraction of agglomerated CNTs was low, which anticipated low electrical percolation value in CNTs/PEI filaments. This numeric model was consistent with SEM and TEM observations, pointing out well-dispersed CNT composition.

Fig. 6 and Table 2 show the electrical conductivity depending on CNT concentration. At a lower wt. \% concentration of CNTs, the conductivity increased drastically with increased CNT content. At a concentration of $0.1 \mathrm{wt}$. $\%$ CNTs, a remarkable rise in the conductivity was noted, to the order of around $10^{9} \mathrm{~S} / \mathrm{cm}$, as a consequence of the built interconnected network of CNTs: in other words, when the system reached its electrical percolation threshold. Overall, the results suggest that at this concentration, when the interconnecting CNTs formed a conductive pathway for electrons due to a large number of electrons flowing, higher electrical conductivity was recorded. Above $0.1 \mathrm{wt} . \%$ CNTs, a moderate increase in electrical conductivity in relation to the concentration of CNTs was noted. In the study by Isayev et al. [32], the electrical conductivity of $10 \mathrm{wt} . \%$ CNTs/PEI samples were noted around $10^{-5} \mathrm{~S} / \mathrm{cm}$ for all the treated and untreated CNT-reinforced PEI nanocomposites. Compared to this reference study [32], here the separation distance between CNTs in the PEI matrix was found to be shorter. This led to an efficient electron flow even at a lower concentration of CNTs. Furthermore, supported by electrical percolation theory [36, 37], estimating the minimum amount of conductive nanofiller $\left(\phi_{c}\right)$ to boost composite conductivity, the percolation threshold was calculated in the range of 0.05 and 0.13 vol. \% CNTs in PEI matrix, whereas in previous studies, $\phi_{c}$ had been around 0.37 vol. \% for CNTs/PLA [8] and 0.27 vol. \% for CNTs/PBI nanocomposites [38]. Thus, the lower percolation threshold depended heavily on the physical geometries of CNTs, as well as their homogeneous and uniform dispersion in the PEI matrix. 


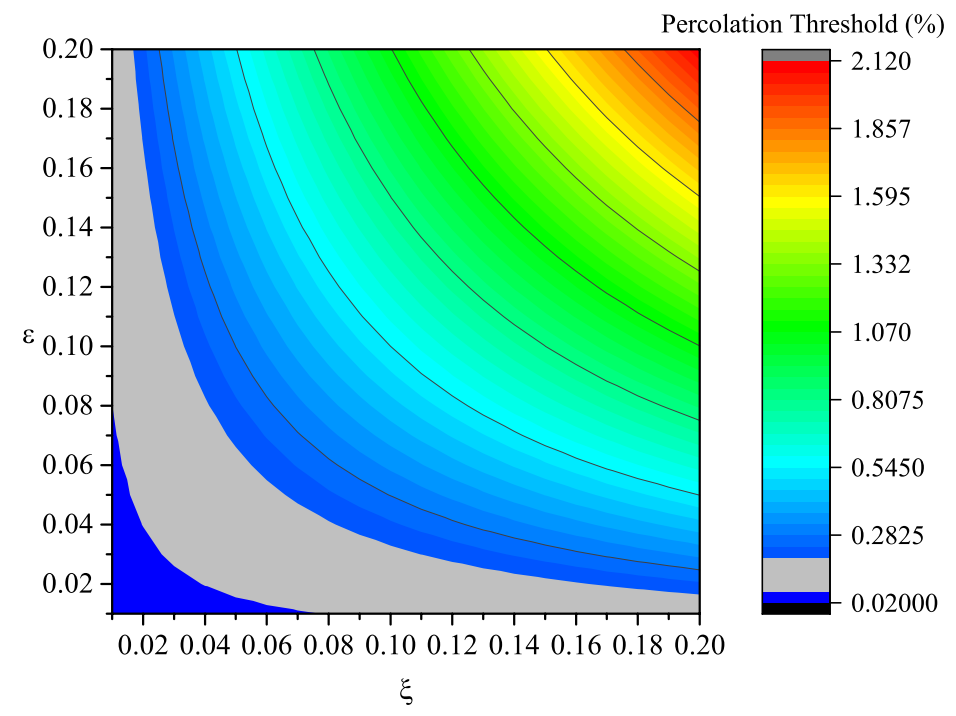

Figure 5: Percolation threshold values by $\epsilon$ and $\xi$ for a nanocomposite that comprises CNTs with an aspect ratio of 30. The gray zone represents the range of the percolation threshold determined using conductivity values of CNTs/PEI nanocomposites.

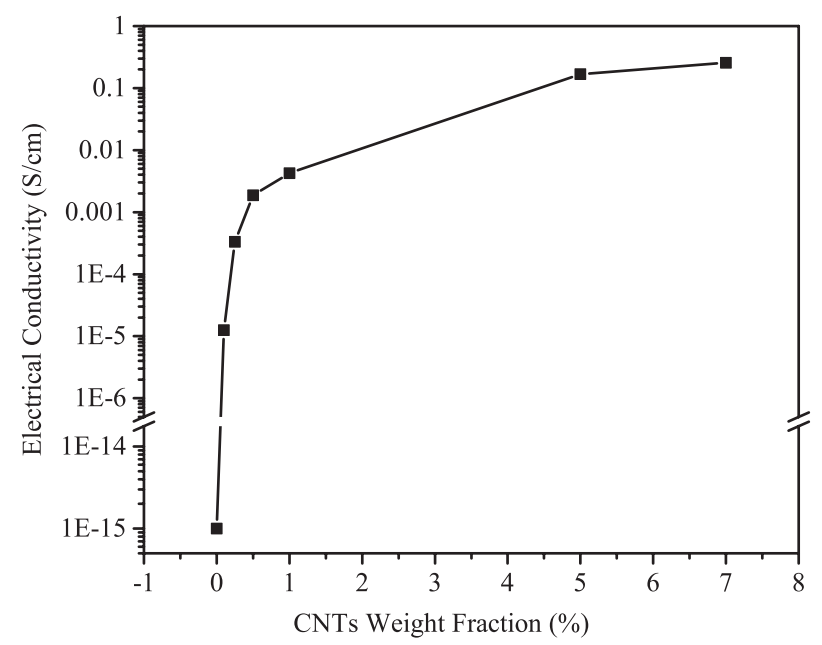

Figure 6: Electrical conductivity of the filaments with respect to CNTs weight fraction with broken y-axis. The percolation threshold for CNTs/PEI was determined above 0.1 wt. \% CNTs in PEI.

\begin{tabular}{ccc} 
Table 2: Surface conductivities of CNTs/PEI filaments at different CNTs weight fraction \\
\cline { 2 - 3 } CNTs wt. \% & CNTs vol. \% & Surface conductivity $(\mathrm{S} / \mathrm{cm})$ \\
\hline 0 & 0 & $10^{-15}$ \\
0.1 & 0.07 & $1.25 \times 10^{-15}$ \\
0.25 & 0.18 & $1.67 \times 10^{-5}$ \\
0.5 & 0.35 & $1.77 \times 10^{-3}$ \\
2 & 1.42 & $4.23 \times 10^{-3}$ \\
5 & 3.58 & $1.69 \times 10^{-1}$ \\
7 & 5.04 & $2.57 \times 10^{-1}$ \\
\hline
\end{tabular}

Figs. 7 a-e demonstrate the complex viscosity, $\tan \delta$, storage, and loss moduli as a function of frequency and van Gurp-Parmen plot for neat PEI, 0.1, 0.25 and 5 wt. \% CNTs/PEI filaments, respectively. The complex viscosities 

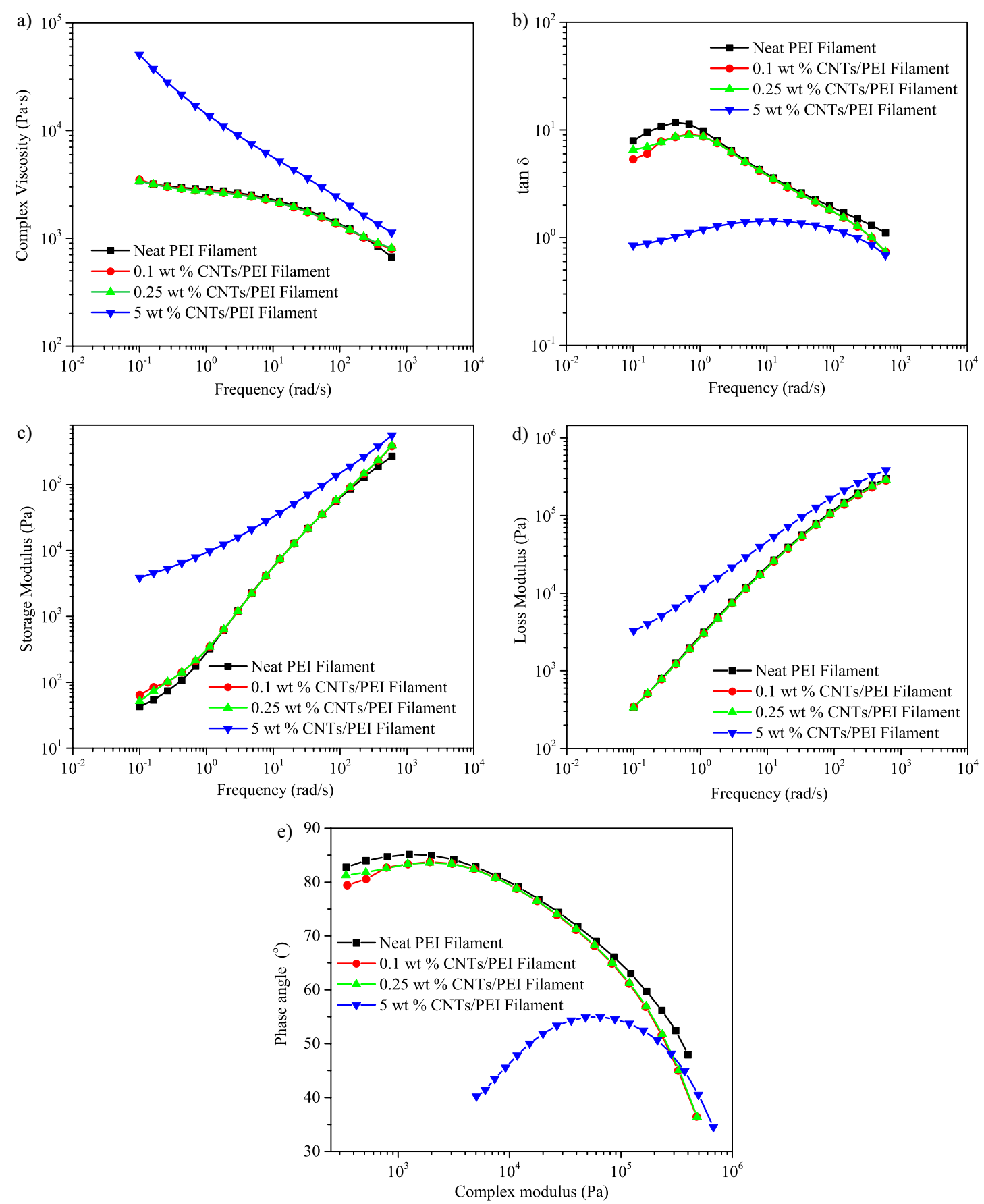

Figure 7: a) Complex viscosity, b) tan $\delta$, c) storage modulus d) loss modulus as a function of frequency e) van Gurp-Parmen plot for neat PEI and CNTs/PEI filaments at different nanotubes loadings. The rheological percolation of the filaments found above $0.25 \mathrm{wt} \% \mathrm{CNTs} / \mathrm{PEI}$.

of both CNTs/PEI and neat PEI filaments are shown in Fig. 7a. Neat PEI, 0.1 and 0.25 wt. \% CNTs/PEI filaments, all showed frequency-dependent shear-thinning behavior. At low frequencies, $5 \mathrm{wt}$ \% CNTs/PEI exhibited viscosity that was one order of magnitude higher than that of a neat PEI system, while showing strong shear-thinning behavior. The effect of CNT concentration became particularly apparent at low frequencies, due to the existence of CNT clusters, and this relative effect diminished when frequency increased with increased shear rates. This behavior was 
a characteristic flow of CNT-suspended solutions: shear forces assisted in the breaking of CNT clusters and eased flow as a result [19]. Thus, higher frequencies led to lower shear viscosity and finally made the system easier to process. This phenomenon was also supported by the decrease in $\tan \delta$ in terms of increased frequency, as shown in Fig. 7b. Similarly, CNT-suspended PEI also exhibited frequency-dependent storage and loss moduli, which was notable, especially in a low-concentration regime (Fig. 7k and 7d). As CNT concentration was increased, the magnitude of both the storage and the loss moduli increased, but their relative fraction decreased [17, 39, 40]. The presence of CNTs disturbed chain relaxation, and energy dissipation was much clearer at low frequencies: as in $0.01-10 \mathrm{rad} / \mathrm{s}$, CNTs addition caused lower $\tan \delta$ than neat PEI systems. At 5 wt. \% CNTs loading, plateau-like steady behavior also indicated widely viscoelastic behavior [17]. This transition from liquid dominant to solid dominant states occurred between the concentration of 0.25 wt. $\%$ and 5 wt. $\%$ CNTs/PEI. Semi-logarithmic plots of phase angle, $\delta$ vs. complex modulus, also known as van Gurp-Parmen plots, revealed the structural differences between the neat matrices and particle-filler integrated systems. As shown in Fig. 7p, the neat PEI and CNTs/PEI incorporating low nanotube concentrations approached a phase angle of $c a .85^{\circ}$, which also emphasized the dominant viscous behavior, while at 5 wt. \% CNTs/PEI filaments, the elastic response became evident in the phase angle and exhibited $c a$. $35^{\circ}$. Overall, this suggested that well-dispersed high-aspect ratio nanotubes could facilitate rheological percolation at lower concentrations than those of the storage modulus [17]. Furthermore, on contrary to some polymer-CNT dispersed states [40], rheological percolation of CNTs/PEI is higher than their electrical percolation. This indicated that CNT-CNT distance is less than a few $\mathrm{nm}$, and the electrical percolation threshold is reached when the first infinite conductive path is formed however the rheological percolation is not reached until a fully-established CNT network is built which also was attributed to "rigid" rheological threshold [41].

Even though this study attempts to provide an understanding of material processing for conductive high-performance $3 \mathrm{D}$ printer filaments, the mechanical properties of these materials have also been one of our design concerns while tuning material compositions. The tensile behavior of these FFF filaments was summarized in Table 3 , and their representative stress/strain graph was demonstrated in Fig. 8. The results pointed out that 5 wt. \% CNTs/PEI filaments had $55 \%$ higher Youngs moduli than neat PEI filament. This increase in stiffness depended heavily on the interfacial shear strength, the aspect ratio of CNTs and their dispersion state, as well as the degree of alignment [25]. The interfacial shear strength, which defined the maximum stress transfer from polymer to CNTs, relied on the polymer-CNTs interface. The reference studies provided landmarks in the literature, highlighting that the tensile strength of filaments could be enhanced by the addition of CNTs up to $10 \%$, as a projection of effective adhesiveness between the polymer and the nanofiller, as well as the homogeneous dispersion and orientation of CNTs in the polymer matrix [25]. 5 wt. $\%$ CNT-reinforced PEI filaments exhibited a slight reduction in the mean tensile strength within the standard error of neat PEI filaments. More remarkably, elongation at break \% of neat PEI filaments was substantially higher than that of 5 wt. \% CNTs/PEI filaments. Thus the decrease in elasticity was an inevitable product of the trade-off between introducing stiff CNTs and decreasing the amorphous PEI composition in nanocomposites [10]. Overall, the results suggest that the incorporation of CNTs into PEI systems enabled stronger but more brittle filament production, which is still comparable with the commercially available products on the market.

Table 3: Quasi-static tensile properties of PEI and CNTs/PEI filaments

\begin{tabular}{cccc}
\hline Filament type & Stiffness (GPa) & Tensile Strength (MPa) & Elongation at break (\%) \\
\hline Neat PEI filament & $2.13 \pm 0.6$ & $93 \pm 1.7$ & $20 \pm 4$ \\
5 wt. \% CNTs/PEI filament & $3.31 \pm 0.13$ & $87 \pm 3$ & $7 \pm 3$ \\
\hline
\end{tabular}

\section{Conclusion}

In this study, the fabrication of conductive CNTs/PEI filaments has been systematically described, and the structureproperty relation of the filaments is revealed through microscopy and thermal and rheological analysis by addressing the resultant properties, including electrical conductivity and tensile strength. Neat PEI and CNTs/PEI filaments at different CNT loadings ranging from 0.1 to $7 \mathrm{wt}$. \% were melt-compounded by a custom-built extrusion process to transfer superior properties of CNTs into additive manufacturing feedstock materials. DSC and TGA analysis showed the fingerprint of CNTs on thermally driven phenomena, where CNT loading, even up to 7 wt. \% did not change the 

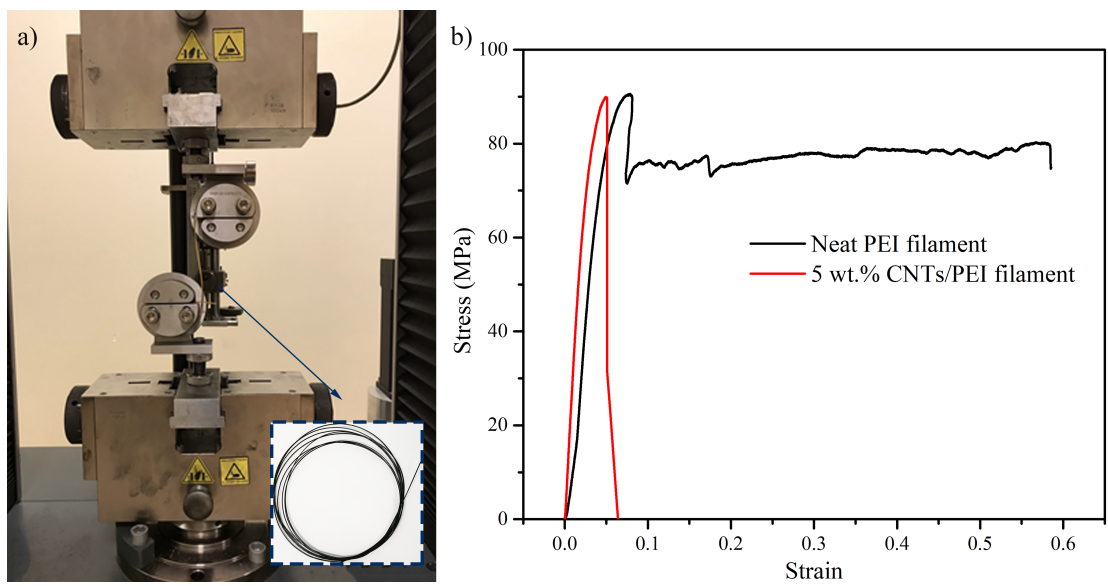

Figure 8: a) Tensile test with single bollard self-tightening type grids and the framed area shows fabricated CNTs/PEI filament sample b) Representative stress-strain graph of neat PEI and 5 wt. \% CNTs/PEI filaments.

kinetic thermal transition as $T g$, and the thermal decomposition of PEI shifted slightly towards higher temperatures within the introduction of CNTs. Morphological characterizations by SEM and TEM demonstrated that CNTs were well dispersed in the PEI matrix without any trace of agglomerates, and oriented due to a sequence of extrusion processes. However, as measured by TEM micrographs, CNT length was significantly reduced to $305 \pm 20 \mathrm{~nm}$ due to the applied high shear forces and mechanical agitation during melt processing. The rheological percolation was found to be above 0.25 wt. \% CNTs/PEI, which was higher than the electrical percolation $(0.1 \mathrm{wt}$. \%). The continuous conductive pathway formed at a lower CNT concentration; however, rheological percolation required a fully intersected CNT network to reach saturation. The incorporation of CNTs enhanced the electrical conductivity of PEI filaments by an order of magnitude of 14 , and the highest electrical conductivity was noted as $2.57 \times 10^{-1} \mathrm{~S} / \mathrm{cm}$ in $7 \mathrm{wt}$. \% CNTs/PEI filament. Compared to neat PEI filaments, the tensile modulus was improved by 55\% by introducing $5 \mathrm{wt}$. \% CNTs; however, this also led to a decrease of $65 \%$ in elongation at break.

\section{Acknowledgment}

The authors are thankful for the Boeing Global Co. project numbered 2016000269 for financial support. The authors thank TUBITAK 3501 (Project No: 116M427) for the postgraduate student scholarship support for Ozge Kaynan. The authors would also like to thank Dr. Gülcan Çorapçıŏlu and Barış Emre Kıral in SUNUM, Sabancı University for TEM and mechanical characterizations, and Assoc. Prof. Güralp Özkoç at Kocaeli University for their characterization supports.

\section{References}

[1] A. D. B. Ferreira, P. R. Novoa, A. T. Marques, Multifunctional material systems: a state-of-the-art review, Composite Structures 151 (2016) 3-35.

[2] M. Naebe, K. Shirvanimoghaddam, Functionally graded materials: A review of fabrication and properties, Applied Materials Today 5 (2016) $223-245$.

[3] G. H. Loh, E. Pei, D. Harrison, M. D. Monzon, An overview of functionally graded additive manufacturing, Additive Manufacturing 23 (2018) $34-44$.

[4] P. Parandoush, D. Lin, A review on additive manufacturing of polymer-fiber composites, Composite Structures 182 (2017) $36-53$.

[5] G. Gomez-Gras, R. Jerez-Mesa, J. A. Travieso-Rodriguez, J. Lluma-Fuentes, Fatigue performance of fused filament fabrication pla specimens, Materials \& Design 140 (2018) 278 - 285.

[6] G. W. Melenka, B. K. Cheung, J. S. Schofield, M. R. Dawson, J. P. Carey, Evaluation and prediction of the tensile properties of continuous fiber-reinforced 3d printed structures, Composite Structures 153 (2016) 866-875.

[7] K. R. Hart, E. D. Wetzel, Fracture behavior of additively manufactured acrylonitrile butadiene styrene (ABS) materials, Engineering Fracture Mechanics 177 (2017) 1-13.

[8] G. Postiglione, G. Natale, G. Griffini, M. Levi, S. Turri, Conductive 3D microstructures by direct 3D printing of polymer/carbon nanotube nanocomposites via liquid deposition modeling, Composites Part A: Applied Science and Manufacturing 76 (2015) $110-114$. 
[9] D. Schmitz, L. Ecco, S. Dul, E. Pereira, B. Soares, G. Barra, A. Pegoretti, Electromagnetic interference shielding effectiveness of abs carbonbased composites manufactured via fused deposition modelling, Materials Today Communications 15 (2018) 70 - 80.

[10] S. Dul, L. Fambri, A. Pegoretti, Filaments production and fused deposition modelling of abs/carbon nanotubes composites, Nanomaterials $8(1)$.

[11] K. C. Chuang, J. E. Grady, R. D. Draper, E.-S. E. Shin, C. Patterson, T. D. Santelle, Additive manufacturing and characterization of Ultem polymers and composites, The Composites and Advanced Materials Expo CAMX, 2015.

[12] S. Berretta, R. Davies, Y. Shyng, Y. Wang, O. Ghita, Fused Deposition Modelling of high temperature polymers: Exploring CNT PEEK composites, Polymer Testing 63 (2017) 251-262.

[13] I. V. Polyakov, G. V. Vaganov, V. E. Yudin, E. M. Ivan'kova, E. N. Popova, V. Y. Elokhovskii, Investigation of properties of nanocomposite polyimide samples obtained by fused deposition modeling, Mechanics of Composite Materials 54 (1) (2018) 33-40.

[14] S. Ghose, K. A. Watson, D. M. Delozier, D. C. Working, J. W. Connell, J. G. Smith, Y. P. Sun, Y. Lin, Thermal Conductivity of Ultem $^{\mathrm{TM}} /$ Carbon Nanofiller Blends, in: Multifunctional Nanocomposites, Vol. 2006, ASME, 2006, pp. $237-245$.

[15] H. Wu, M. Sulkis, J. Driver, A. Saade-Castillo, A. Thompson, J. H. Koo, Multi-functional ULTEM $\notin 1010$ composite filaments for additive manufacturing using Fused Filament Fabrication (FFF), Additive Manufacturing 24 (2018) 298-306.

[16] J. Gonçalves, P. Lima, B. Krause, P. Pötschke, U. Lafont, J. Gomes, C. Abreu, M. Paiva, J. Covas, Electrically conductive polyetheretherketone nanocomposite filaments: from production to fused deposition modeling, Polymers 10 (8) (2018) 925.

[17] D. Bangarusampath, H. Ruckdäschel, V. Altstädt, J. K. Sandler, D. Garray, M. S. Shaffer, Rheology and properties of melt-processed poly (ether ether ketone)/multi-wall carbon nanotube composites, Polymer 50 (24) (2009) 5803-5811.

[18] T. Liu, Y. Tong, W.-D. Zhang, Preparation and characterization of carbon nanotube/polyetherimide nanocomposite films, Composites science and technology 67 (3-4) (2007) 406-412.

[19] E. Özden-Yenigün, Y. Menceloğlu, M. Papila, Mwcnts/p (st-co-gma) composite nanofibers of engineered interface chemistry for epoxy matrix nanocomposites, ACS Applied Materials \& Interfaces 4 (2) (2012) 777-784.

[20] M. Tunckol, E. Z. Hernandez, J.-R. Sarasua, J. Durand, P. Serp, Polymerized ionic liquid functionalized multi-walled carbon nanotubes/polyetherimide composites, European Polymer Journal 49 (12) (2013) 3770-3777.

[21] S. Yang, J. R. Castilleja, E. Barrera, K. Lozano, Thermal analysis of an acrylonitrile-butadiene-styrene/swnt composite, Polymer Degradation and Stability 83 (3) (2004) 383-388.

[22] S. Koutsoumpis, P. Klonos, K. N. Raftopoulos, C. M. Papadakis, D. Bikiaris, P. Pissis, Morphology, thermal properties and molecular dynamics of syndiotactic polystyrene (s-ps) nanocomposites with aligned graphene oxide and graphene nanosheets, Polymer 153 (2018) $548-557$.

[23] X. Yan, H. Dong, Z. Xiao, Y. Liu, T. Liu, H. G. Chae, S. Kumar, Effect of high-shear mixing by twin-screw extruder on the dispersion and homogeneity of polyacrylonitrile/carbon nanotube composite solution, Polymer Composites 38 (4) (2017) $719-726$.

[24] J.-Y. Lee, J. An, C. K. Chua, Fundamentals and applications of 3d printing for novel materials, Applied Materials Today 7 (2017) $120-133$.

[25] M. K. Pitchan, S. Bhowmik, M. Balachandran, M. Abraham, Effect of surface functionalization on mechanical properties and decomposition kinetics of high performance polyetherimide/mwent nano composites, Composites Part A: Applied Science and Manufacturing 90 (2016) $147-160$.

[26] M. Hegde, E. T. Samulski, M. Rubinstein, T. J. Dingemans, The role of crystallinity in swcnt-polyetherimide nanocomposites, Composites Science and Technology 110 (2015) 176-187.

[27] L. F. P. Santos, B. Ribeiro, L. R. O. Hein, E. C. Botelho, M. L. Costa, Processing, thermal and mechanical behaviour of PEI/MWCNT/carbon fiber nanostructured laminate, Materials Research Express 4 (11) (2017) 115037.

[28] K. Mittal, Polyimides: Synthesis, Characterization, and Applications, no. v. 1, Springer US, 2013.

[29] K. Hamad, M. Kaseem, H. Yang, F. Deri, Y. Ko, Properties and medical applications of polylactic acid: A review., Express Polymer Letters 9 (5) (2015) 435-455.

[30] I. González, J. Eguiazabal, Widely dispersed pei-based nanocomposites with multi-wall carbon nanotubes by blending with a masterbatch, Composites Part A: Applied Science and Manufacturing 53 (2013) 176-181.

[31] F. V. Ferreira, W. Francisco, B. R. C. de Menezes, L. D. S. Cividanes, A. dos Reis Coutinho, G. P. Thim, Carbon nanotube functionalized with dodecylamine for the effective dispersion in solvents, Applied Surface Science 357 (2015) 2154-2159.

[32] A. Isayev, R. Kumar, T. M. Lewis, Ultrasound assisted twin screw extrusion of polymer-nanocomposites containing carbon nanotubes, Polymer 50 (1) (2009) 250-260.

[33] M. K. Pitchan, S. Bhowmik, M. Balachandran, M. Abraham, Process optimization of functionalized mwcnt/polyetherimide nanocomposites for aerospace application, Materials \& Design 127 (2017) 193-203.

[34] R. Socher, B. Krause, M. T. Müller, R. Boldt, P. Pötschke, The influence of matrix viscosity on mwcnt dispersion and electrical properties in different thermoplastic nanocomposites, Polymer 53 (2) (2012) 495-504.

[35] J. Li, P. C. Ma, W. S. Chow, C. K. To, B. Z. Tang, J.-K. Kim, Correlations between percolation threshold, dispersion state, and aspect ratio of carbon nanotubes, Advanced Functional Materials 17 (16) (2007) 3207-3215.

[36] G. Pike, C. Seager, Percolation and conductivity: A computer study. i, Physical review B 10 (4) (1974) 1421.

[37] C. Seager, G. Pike, Percolation and conductivity: A computer study. ii, Physical Review B 10 (4) (1974) 1435.

[38] K. Gnanasekaran, T. Heijmans, S. Van Bennekom, H. Woldhuis, S. Wijnia, G. de With, H. Friedrich, 3d printing of cnt-and graphene-based conductive polymer nanocomposites by fused deposition modeling, Applied Materials today 9 (2017) 21-28.

[39] J. Zhong, A. I. Isayev, Properties of polyetherimide/graphite composites prepared using ultrasonic twin-screw extrusion, Journal of Applied Polymer Science 132 (5) (2015) 41397.

[40] P. Pötschke, T. Fornes, D. Paul, Rheological behavior of multiwalled carbon nanotube/polycarbonate composites, Polymer 43 (11) (2002) 3247-3255.

[41] C. Penu, G.-H. Hu, A. Fernandez, P. Marchal, L. Choplin, Rheological and electrical percolation thresholds of carbon nanotube/polymer nanocomposites, Polymer Engineering \& Science 52 (10) (2012) 2173-2181. 